\title{
Emphasizing
}

Mental Health in

Nursing Education

BY SUE CAVANAUGH

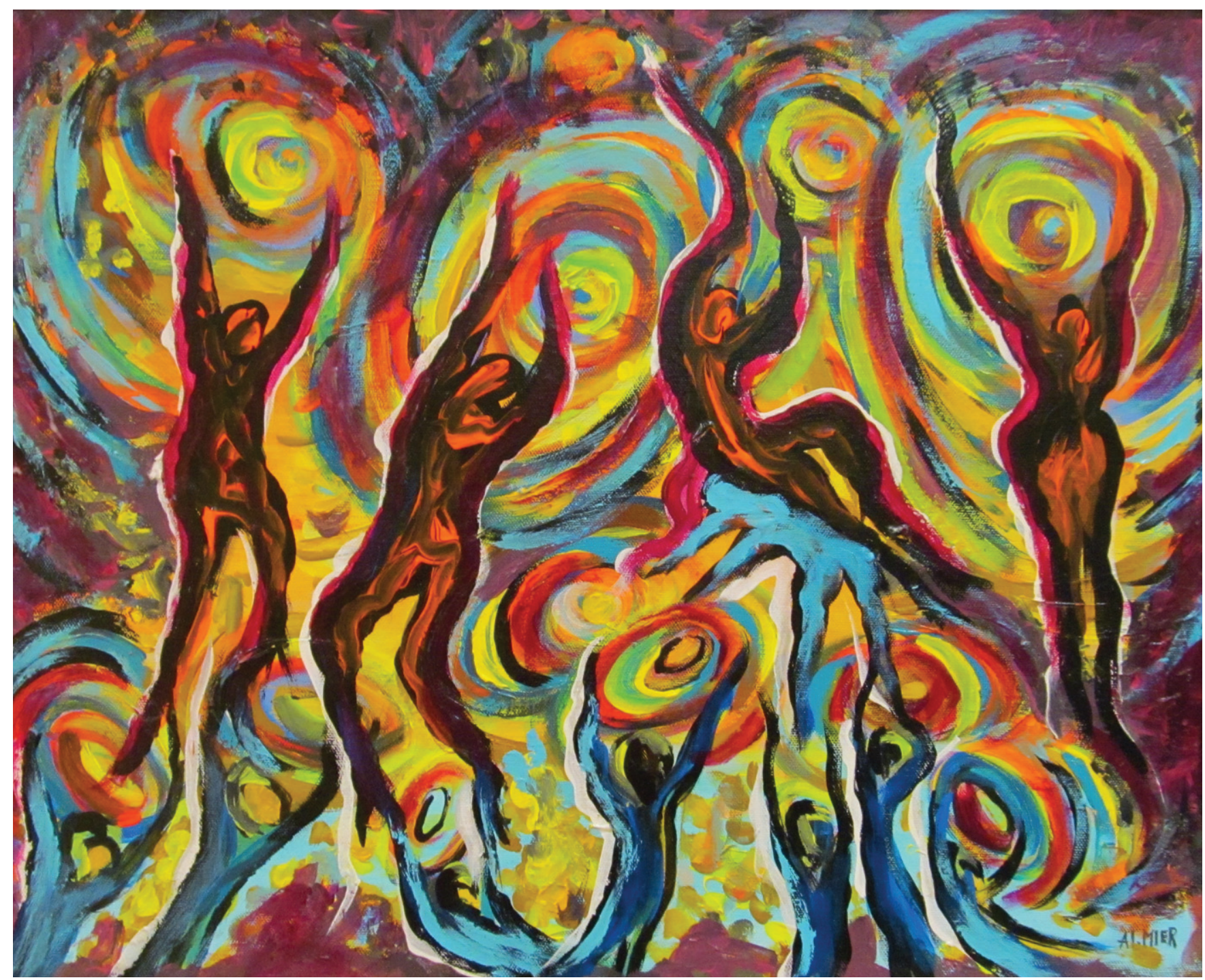

\section{Journey to Enlightenment}

"In spite of having challenges with mental health concerns, I look for the joy and fulfilment in life. Reaching up for the passion and enthusiasm in life, I see colours and potential in others and the art that I create." 
B ecoming a psychiatric mental health $(\mathrm{PMH})$ nurse wasn't even on Natasha Churchill's radar when she started nursing school. Her plan was to become a surgical nurse. Then she started her mental health rotation and something clicked. "On the first day, I thought, 'Oh my gosh, this is what I want!'” After graduation, Churchill worked in mental health acute care for five years and is now a sessional faculty member at the Centre for Nursing Studies in St. John's, where she facilitates mental health clinical placements.

Churchill's decision to pursue a career in $\mathrm{PMH}$ nursing is not that common. A 2012 Canadian Institute for Health Information report on current trends in nursing practice found that only 5.7 per cent of all regulated nurses - RNs and nurse practitioners, licensed practical nurses and registered psychiatric nurses (RPNs) - working in direct care in Canada were employed in psychiatric mental health.

The low percentage may reflect the fact that students are often not even exposed to $\mathrm{PMH}$ nursing in their education. According to a 2006 environmental scan of nursing schools across the country, conducted by the Canadian Federation of Mental Health Nurses (CFMHN), 20 per cent of undergraduate programs did not offer a stand-alone course in $\mathrm{PMH}$ nursing or provide their students with any mental health clinical experience.

In a 2009 position paper, CFMHN called for mandatory mental health education that includes theory and clinical work for all undergraduate nursing students. According to the document, "Nurses who graduate without any clinical experience in $\mathrm{PMH}$ nursing state that they have insufficient knowledge, skills and confidence in intervening with persons who have mental health problems and mental illnesses." The paper cites research showing that "many nurses are unprepared to support the mental health needs of persons with mental health problems and mental illnesses."

Stigma about working in the mental health system persists, due in large part to popular media portrayals of PMH nurses as cold and controlling autocrats - most famously in One Flew Over the Cuckoo's Nest. The film, now almost 40 years old, is still the first thing many students mention when asked what comes to mind when they think of mental health nursing, according to Judy Osborne, a clinical faculty member at Trent University. "During orientation, we spend a great deal of time talking about stigma and misperceptions about mental health nursing," she says.

In the four western provinces, registered psychiatric nursing is a distinct regulated health profession, with its own national code of ethics and standards of practice, as well as separate education programs that focus exclusively on PMH nursing. "RPNs are very well-versed and knowledgeable," says Bernadine Wojtowicz, an instructor at the University of Lethbridge. "If there is an RPN staff nurse at my students' clinical placement, I encourage them to use that individual as a resource."

In nursing schools that do offer courses in psychiatric mental health, there is substantial variation in length and format. Some courses last an entire semester; others may be as short as six weeks. While there is no standardized curriculum, courses generally consist of theory classes and clinical placements. Topics covered range from the use of

\section{"There is no health without mental health"}

Louise Bradley, RN, president and CEO of the Mental Health Commission of Canada, firmly believes mental health nursing is critically important throughout every discipline of the profession. "We need to truly look at mental health as part of all nursing - specialty nursing in particular. The links between physical and mental health are strong."

People with serious mental illness frequently have co-existing chronic physical conditions. Similarly, people with chronic physical conditions are at risk of developing mental health problems, which can, in turn, impede their ability to self-care. According to the Canadian Mental Health Association, people living with chronic physical health conditions experience depression and anxiety at twice the rate of the general population.

Bradley, who has practised mental health nursing in all her nursing roles, cites core competencies in mental health as a foundational part of nursing. "Surgical nursing does not need to be part of mental health nursing, but mental health training should be part of surgical nursing."

A steering committee made up of physician and mental health organizations, including the MHCC, is in the process of developing mental health-related goals, principles and core competencies for physicians. The intention is to recognize and address the interactions between physical and mental health, provide practice support, improve access to mental health services, ameliorate the experience of care, and reduce stigma surrounding mental health issues. 


\section{The MHCC knowledge exchange}

\author{
The MHCC regularly hosts \\ webinars on a variety of mental \\ health issues. Webinar topics have \\ included the link between prevent- \\ able injury and mental health, and \\ technology to assist in suicide \\ prevention. Following each webinar, \\ a recording and a PDF copy of the \\ PowerPoint presentation are made \\ available for free on its website. \\ You can sign up for future webinars \\ by e-mailing your interest to \\ info@mentalhealthcommission.ca. \\ The MHCC also hosts a training \\ program on putting research into \\ action, bringing together dozens of \\ participants for two days of on-site \\ knowledge exchange training with \\ internationally respected profes- \\ sionals, followed by the implementa- \\ tion of the participants' knowledge \\ translation projects with the support \\ of mentors, online training sessions \\ options are available for nurses \\ who would like to increase their \\ knowledge of mental health issues. \\ Among them: \\ - Brandon University has a master \\ of psychiatric nursing program, \\ the only one of its kind in \\ Canada. \\ - CNA offers certification in \\ psychiatric and mental health \\ nursing. As of the end of 2012 , \\ there were more than 1,600 \\ nurses certified in this specialty. \\ - Mental Health First Aid Canada \\ offers training to help people \\ identify mental health problems \\ and provide initial help. More \\ than 90,000 Canadians have \\ been trained since 2007. In 2013, \\ the Ordre des infirmières et \\ infirmiers du Québec recognized \\ mental health first aid as a \\ continuing education course.
} and a community of practice. Applications for the 2015 SPARK Training Institute will be available on the MHCC website this fall.

assessment tools and the therapeutic relationship and its boundaries to specific conditions and diagnoses and their symptoms and treatment. Osborne, who teaches Trent's clinical component in the acute mental health inpatient unit of a local hospital, says she also stresses safety guidelines and expectations with respect to professionalism and unit procedures.

Stigma is another important topic. During clinical placements, faculty make sure they immediately address any bias they see students exhibiting toward clients. "Students must reflect on their own attitudes and beliefs about mental health," says Churchill. In her experience,

\section{Post-graduate resources}

Post-baccalaureate educational

by the time a clinical rotation is over, students are more aware not only of their own attitudes toward mental illness, but also of stigma in social media and popular culture.

Clinical placements vary, depending on what facilities are available in the region. Students might be assigned to an acute care ward, a community health centre or a rehabilitation centre. In some programs, they might visit multiple locations during their placement period. Osborne augments her students' clinical experience with a couple of afternoon field trips. One is to a homeless shelter, where students assist in preparing and serving dinner; another is to a women's shelter, where they learn about the referral process for abused women and gain a better understanding of the concomitant mental health issues that victims of domestic violence experience, as well as how to screen effectively for abuse.

One of the key strengths PMH nurses must have, observes Wojtowicz, is the ability to look past a diagnosis and see the client as a person first. "They need to learn how to get out of the medical model and focus on the person's holistic needs," she explains. "Students are often looking for a quick fix, but you can't slap a cast on a mental health diagnosis."

Sherri Melrose is an associate professor at Athabasca University who has worked and taught in the field of mental health for several decades. Although she encourages her students to consider PMH nursing as a career, she believes even those who choose other paths need to learn as much as they can about the mental health conditions they're likely to come across in their field. For example, a nurse working in public health will almost certainly have clients who are experiencing postpartum depression; an emergency nurse may treat an injured patient who reveals that the "accident" was actually a suicide attempt. Knowing what to do in those situations is critical, Melrose says.

Wojtowicz agrees. "Whether they are dealing with an anxiety attack, a psychotic break due to drug use, or a parent's grief, nurses are going to encounter mental health issues, no matter where they work."

SUE CAVANAUGH IS A FREELANCE WRITER IN OTTAWA. 